\title{
Bend- and Twist-Insensitive Flexible Multimode Polymer Optical Interconnects
}

\author{
Nikolaos Bamiedakis, Fengyuan Shi, Richard V. Penty, Senior Member, IEEE, \\ Ian H. White, Fellow, IEEE and Daping Chu, Senior Member, IEEE
}

\begin{abstract}
Polymer multimode optical waveguides can enable high-speed short-reach optical interconnection at low cost within high performance electronic systems. The formation of such waveguides on flexible substrates can offer important additional advantages such as light weight, ability to be tightly bent and reconfigurability which are particularly important in environments where space, weight and shape conformity are critical, for instance in vehicles and aircraft. The ability of such flexible optical interconnects to be tightly bent and twisted with low excess loss is crucial in enabling their use in systems with limited space and with movable parts. As a result, in this work, we present a new design of such flexible polymer multimode waveguides that achieves improved bending loss performance over the conventional waveguide design. It is experimentally shown that the proposed design achieves a very low excess loss of $0.5 \mathrm{~dB}$ for a $3 \mathrm{~mm}$ radius bend under a $50 \mu \mathrm{m}$ MMF launch. In comparison, flexible waveguides with the conventional design exhibit a $2 \mathrm{~dB}$ excess loss under the same launch and bend conditions. Additionally, useful rules that associate the twisting loss performance of flexible polymer waveguide samples with their geometric characteristics are derived. It is shown that negligible twisting losses ( $<0.1 \mathrm{~dB}$ for a $50 \mu \mathrm{m}$ MMF input) can be achieved when the dimensions of the waveguide samples are appropriately selected. The results demonstrate the strong potential of such bend- and twist-insensitive flexible polymer waveguides for use in next-generation vehicles and aircraft.

Index Terms - optical interconnects, polymer waveguides, multimode waveguides, flexible substrates, waveguide bends, twisting loss performance.
\end{abstract}

\section{INTRODUCTION}

$\mathrm{O}$ ptical interconnects have attracted significant interest for use inside high performance electronic systems, such as data centres and storage systems, as they offer important advantages over their copper-based counterparts when

Manuscript received April xx, 2020; revised July xx, 2020; accepted July xx, 2020. Date of publication February xx, 2020; date of current version July xx, 2020

The authors would like to acknowledge Dow Corning for the provision of the polymer samples and materials through the CAPE OIC Future project, Jaguar Land Rover for their financial support through the CAPE LEASA project and the UK EPSRC for their support through the Seamatics research grant (EP/M015165/1) and the Centre for Doctoral Training in Integrated Photonic and Electronic Systems (EP/L015455/1).

Data related to this publication is available at the University of Cambridge data repository https://doi.org/10.17863/CAM.55074

N. Bamiedakis, F. Shi, R. V. Penty, I. H. White and D. Chu are with the Centre for Photonic Systems, Electrical Engineering Division, Department of Engineering, University of Cambridge, Cambridge CB3 OFA, U.K. (e-mail: nb301@cam.ac.uk)

Color versions of one or more of the figures in this paper are available online at http://ieeexplore.ieee.org.

Digital Object Identifier 10.1109/xxxx operating at high data rates (> $10 \mathrm{~Gb} / \mathrm{s}$ ): larger bandwidth, higher power efficiency and density, and more relaxed thermal management requirements $[1,2]$. Multimode polymer waveguides is a promising technology for achieving optical interconnection at the board level as it allows the costeffective integration of optical waveguides with standard electronics and achieves low loss and high bandwidth onboard optical connections [3-6]. The technology leverages the recent development of polymer materials with the essential thermal, mechanical and optical properties for direct integration onto standard printed circuit boards (PCBs) and the use of optical waveguides with relatively large dimensions (typically in the range of 20 to $70 \mu \mathrm{m}$ ) that offer relaxed alignment tolerances and enable system assembly with conventional pick-and-place tools of the electronics industry [7-9]. In recent years, various polymer-based optical waveguide technologies have been reported and numerous prototype optical backplanes and board-level interconnects have been demonstrated [3, 10-14]. These systems feature large waveguide arrays embedded in rigid PCBs to achieve large aggregate data capacities. Bandwidth studies on multimode waveguides have shown that they can exhibit a bandwidth-length product in excess of $30 \mathrm{GHz} \times \mathrm{m}$ despite their highly multimoded nature $[15,16]$, while data transmission of $40 \mathrm{~Gb} / \mathrm{s}$ and $56 \mathrm{~Gb} / \mathrm{s}$ over a $1 \mathrm{~m}$ long waveguide have been demonstrated using non-return-to-zero (NRZ) [17] and 4-level pulse amplitude (PAM-4) modulation [18] respectively.

Further, the formation of such waveguides on flexible substrates can offer important additional advantages. It enables the formation of versatile thin, lightweight, detachable and bendable optical connections that can be used in a wide range of applications. These attributes are particularly important in environments where space, weight and shape conformity are crucial such as in next-generation vehicles and aircraft. The advent of driverless cars and the associated requirement for high-speed, low-latency communication between a multitude of sensors and imaging cameras located at the car periphery with a central processing unit where realtime decisions are made, pushes the need for low-cost lightweight flexible high-speed communication links. Similarly, detachable flexible optical ribbons can offer shortreach high-speed communication between high performance electronic chips and enable significant improvements in system performance $[2,14]$.

Flexible polymer-based optical interconnection technologies have been reported in recent years [19-28]. Their practical use is however limited by the excess loss induced due to bending and twisting and the minimum associated 
bending radius. In this work therefore, we present a new waveguide design for polymer-based flexible optical interconnects that offers improved bending loss performance over the conventional design. The bending performance of flexible waveguide samples featuring this novel bendinsensitive design is assessed under different launch conditions and it is experimentally shown that significant improvement is achieved over the conventional design. In addition, useful rules that associate the twisting loss performance of flexible waveguide samples with their geometric characteristics are derived and are validated with samples of different geometries. It is shown that negligible excess twisting loss can be achieved if the dimensions of the samples are appropriately selected. The results demonstrate the strong potential of these bend- and twist-insensitive flexible optical interconnects in real-world applications.

The remainder of the paper is structured as follows. Section 2 presents the flexible polymer waveguide technology and the proposed new design, while section 3 reports the characterisation of the bending loss performance of samples with the new and conventional design. In section 4 the twisting loss performance of flexible polymer waveguide ribbons is presented and useful rules that ensure negligible twisting losses are derived. Finally, section 5 concludes the paper.

\section{FLEXIBLE MULTIMODE POLYMER WAVEGUIDES}

The flexible waveguides used in this work are fabricated from siloxane polymer materials developed by Dow Corning: Dow Corning ${ }^{\circledR}$ WG-1020 Optical Elastomer (core) and XX-1023 Optical Elastomer (cladding). These materials have been developed with the essential mechanical, thermal and optical properties for direct integration onto PCBs. They can withstand the high temperatures in excess of $300^{\circ} \mathrm{C}$ required for solder reflow and board lamination, and the standard operating conditions of standard electronics, and exhibit long lifetimes and low material absorption $(\sim 0.04 \mathrm{~dB} / \mathrm{cm})$ at the datacommunication wavelength of $850 \mathrm{~nm}[29,30]$. They can be deposited on both rigid (e.g. FR4, glass, silicon) and flexible (e.g. polyimide) substrate with different methods (spin coating, doctor blading, etc) and patterned with a wide range of techniques such as photolithography, embossing, direct laser writing and direct dispensing $[22,31,32]$. The refractive index (RI) of the core and cladding material is $\sim 1.5255$ and 1.5104 respectively at $850 \mathrm{~nm}$ resulting in a RI difference $\Delta \mathrm{n}$ of $\sim 0.015$. Typically, waveguides with core dimensions $\sim 50 \times 50 \mu \mathrm{m}^{2}$ and a pitch of $250 \mu \mathrm{m}$ are employed as these provide a good match with standard VCSEL arrays and MMF ribbon cables.

The conventional waveguide design consists of buried waveguide core inside a thick layer of cladding material [Fig. 1(a)]. The thickness of the bottom cladding is typically $>20 \mu \mathrm{m}$ to ensure sufficient separation between the waveguide core and substrate and to mask any substrate roughness. Similarly, a thick top cladding layer $(>20 \mu \mathrm{m})$ is applied to embed the waveguide cores and protect them from any dust contamination and external damage. The new waveguide design for improved bending loss performance from flexible samples proposed here is shown in Fig. 1(b).

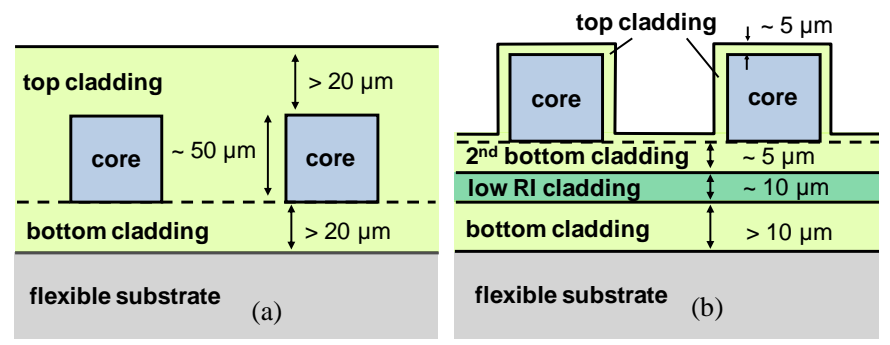

Fig. 1. (a) Conventional and (b) proposed bend-insensitive waveguide design for flexible multimode polymer optical interconnects.

There are two differences between the conventional design and the proposed one: (i) an additional cladding layer from a polymer material with a RI lower than that of the cladding is introduced below the waveguide cores, and (ii) the thickness of the top cladding layer is significantly reduced to a few microns. These two additional features improve the bending loss performance of the waveguides as light is better confined inside the waveguide core when the samples are bent in the out-of-plane direction due to the presence of a material with a lower RI index close to the waveguide core: the air in case of sample bending towards the bottom and the low RI cladding layer for sample bending towards the top. It should be noted that the presence of the thin top cladding and $2^{\text {nd }}$ bottom cladding (right below the waveguide cores) layers is essential to ensure that light propagation inside the waveguide core remains un-affected when the samples are not flexed. Removing these layers would increase the propagation loss due to the larger RI difference between the core and air at the 3 top waveguide sides and the core and lower RI cladding at the waveguide bottom side. Additionally, the bandwidth of the waveguide would be reduced as the RI difference between core and cladding would be increased.

Simulations are carried out using a commercial electromagnetic mode solver (Fimmwave, finite difference method) to obtain the required thickness of the cladding layer that would achieve similar loss and bandwidth performance from the two designs when no flexure is applied. The simulated structure is shown in Fig. 2. The thickness of the top and bottom cladding layer $d$ is varied from $0 \mu \mathrm{m}$ to $14 \mu \mathrm{m}$. The largest value of $14 \mu \mathrm{m}$ emulates the conventional design as the cladding thickness is large enough to isolate the waveguide core from the other two cladding layers (air above and low RI cladding layer below the waveguide core).

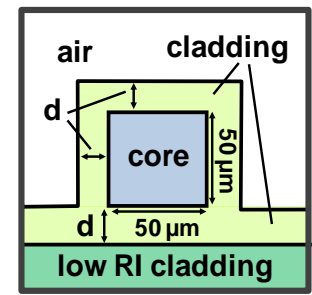

Fig. 2. Simulated waveguide structure.

The waveguide modes are calculated for different values of the cladding thickness $d$ at the $850 \mathrm{~nm}$ wavelength and their effective and group refractive index and confinement factor are obtained. The waveguide with the conventional design $(d=14 \mu \mathrm{m})$ supports $\sim 1080$ guided modes. The reduction of the cladding thickness $d$ results in a gradual increase in the number of supported modes. Fig. 3 shows the group refractive 
index and confinement factor of the waveguide modes for the different cladding thickness values $d$. The latter affects the mode loss while the former the waveguide bandwidth. It can be noticed that for a cladding thickness $d$ of $\sim 5 \mu \mathrm{m}$, very similar group refractive indices and confinement factors are obtained for the large majority of the waveguide modes as in the conventional design $(d=14 \mu \mathrm{m})$. As a result, the target thickness of the cladding layer for the materials and waveguide dimensions used here is chosen to be $\sim 5 \mu \mathrm{m}$. The simulation results also indicate that there is a relatively relaxed fabrication tolerance for the cladding thickness value $d$ as the observed differences in mode parameters are very small for the majority of the guided modes for $d$ values in the range 3 to $7 \mu \mathrm{m}$.

(a)

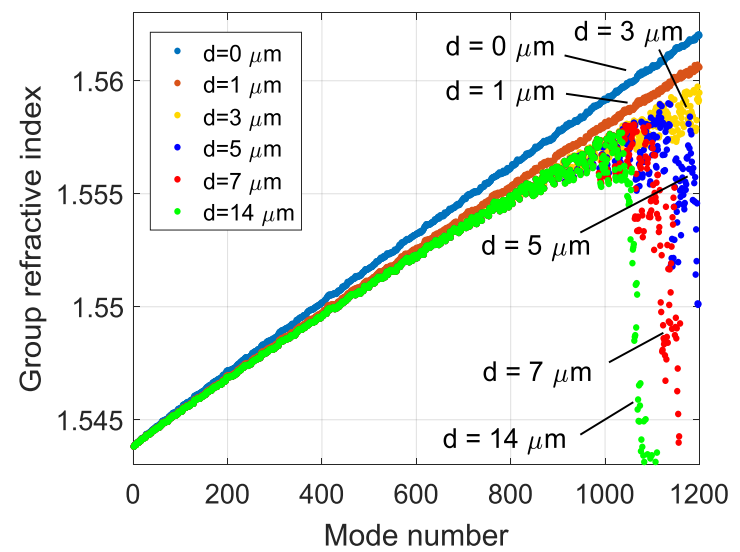

(b)

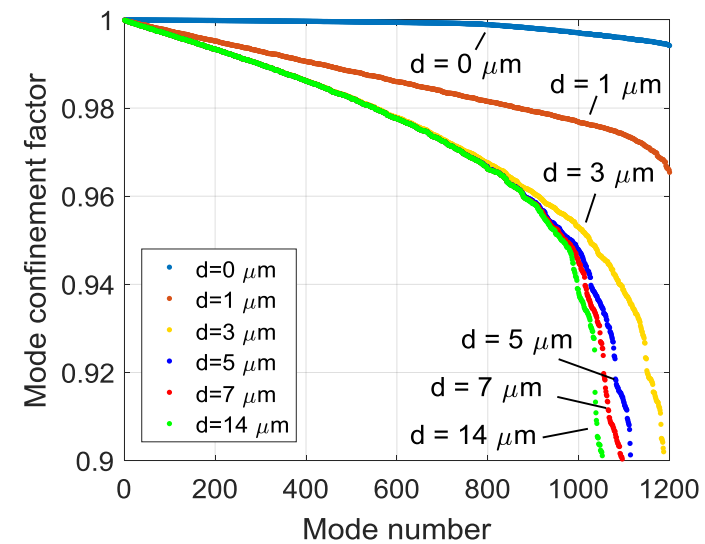

Fig. 3. (a) Group refractive index and (b) confinement factor for the waveguide modes for different values of the cladding thickness $d$.

The two new features of the proposed design can be easily achieved in practice by introducing a couple of additional straightforward steps in the fabrication process of the conventional structure. These include the deposition of the low RI cladding layer and the thin $2^{\text {nd }}$ bottom cladding layer below the waveguide cores. Their thickness, as well as that of the top cladding layer, can be controlled through the deposition method. As suggested by the simulation results presented above, the thickness of the top and bottom cladding layers has relaxed fabrication tolerances, with a variation of the order of $\pm 1 \mu \mathrm{m}$ not expected to severely impact the performance of the waveguides. Fig. 4 summarises the fabrication process for the proposed waveguide design assuming standard photolithography. All planar cladding layers are deposited on the flexible substrate and cured with ultraviolet (UV) light, while the core layer is exposed to UV light via a photomask after deposition to generate the waveguide patterns. The waveguide cores are formed after wet development of the samples, while the thin top cladding is subsequently deposited over the waveguide cores.
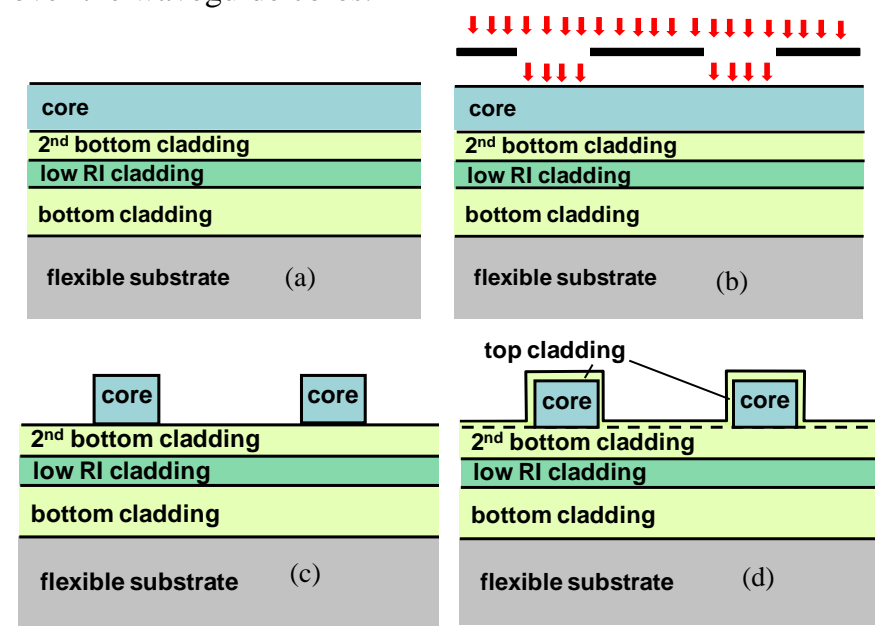

Fig. 4. Summary of the fabrication process of the bend-insensitive flexible polymer waveguides: (a) deposition and curing of the 4 polymer layers on the flexible substrate, (b) UV exposure of the core layer through a photomask, (c) development of the waveguides and (d) deposition of the thin top cladding.

The samples used in this work are fabricated with the process described above using spin coating as the deposition method and photolithography for patterning the waveguide structures. The thickness of the deposited layers is controlled via the spinning speed for each coating step. The substrates used are $125 \mu \mathrm{m}$ thick polyimide sheets, while poly-dimethylsiloxane (PDMS) is used as the material for the low RI cladding layer. Its RI value is $\sim 1.41$ at $850 \mathrm{~nm}$, which is substantially lower than that of the siloxane cladding material $(\sim 1.51$ at $850 \mathrm{~nm})$. In order to improve the adhesion of the $2^{\text {nd }}$ bottom cladding layer on the PDMS layer (low RI cladding layer), short oxygen plasma etching is applied before the deposition of the $2^{\text {nd }}$ bottom cladding layer. Flexible waveguide samples that comprise an array of 12 waveguides $70 \mathrm{~mm}$ in length are produced. The waveguide facets are exposed with a Disco DAD341 dicing saw. No polishing steps are undertaken to improve the quality of the facets produced.

Fig. 5 shows images of the fabricated samples. The deposition of the thin top cladding layer produces smooth cladding tails over the vertical sidewalls of the waveguides [Fig. 5(b)]. Their presence is not expected to affect the bending performance of the waveguides as the flexure results in sample bending in the other direction (out of plane: towards the top or bottom). Flexible waveguide samples based on the conventional design [Fig. 1(a)] are also fabricated with the same materials and process to allow the comparison of the bending performance of the two designs. Table I lists the thickness of the polymer layers for both type of fabricated samples. The top and bottom cladding layers for the samples with the conventional design are made substantially thicker as this improves the mechanical robustness of the samples without affecting the light propagation inside the waveguides. The measured waveguide width is $\sim 53$ and $56 \mu \mathrm{m}$ for the samples with the new and conventional design respectively. 


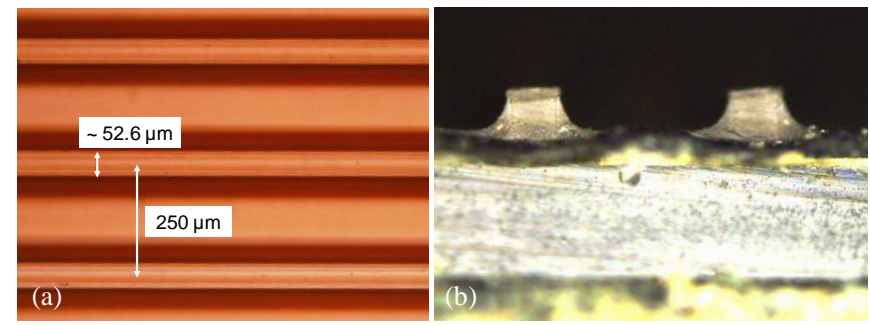

Fig. 5. Images of the fabricated flexible waveguide samples: (a) top view and (b) sample cross section. The smooth tails of the thin top cladding layer can be clearly noticed.

TABLE I

THICKNESS OF POLYMER LAYERS IN FABRICATED SAMPLES

\begin{tabular}{|c|c|c|c|c|c|}
\hline & \multicolumn{5}{|c|}{ Layer thickness $(\mu \mathrm{m}) \pm 1 \mu \mathrm{m}$} \\
\hline Design & $\begin{array}{c}\text { bottom } \\
\text { cladding }\end{array}$ & $\begin{array}{c}\text { low RI } \\
\text { cladding }\end{array}$ & $\begin{array}{c}2^{\text {nd }} \text { bottom } \\
\text { cladding }\end{array}$ & $\begin{array}{c}\text { waveguide } \\
\text { core }\end{array}$ & $\begin{array}{c}\text { top } \\
\text { cladding }\end{array}$ \\
\hline conventional & 92 & -- & -- & 59 & 46 \\
\hline new & 7.8 & 19.3 & 5.1 & 51.2 & 5.8 \\
\hline
\end{tabular}

\section{BENDING LOSS PERFORMANCE}

The bending loss performance of the flexible samples featuring the two designs is assessed under different launch conditions. Due to the highly multimoded nature of the waveguides, their loss performance strongly depends on their mode power distribution inside the waveguide and therefore, the employed launch condition at the waveguide input. Lower order modes are less susceptible to bending loss than higher order modes due to their better light confinement inside the waveguide core. As a result, different launches result in different mode power distributions inside the waveguide and therefore in different bending loss. For the measurements reported here, the launch conditions used include: (a) a 4/125 $\mu \mathrm{m}$ SMF with a numerical aperture (NA) of 0.13 , (b) a 50/125 $\mu \mathrm{m}$ MMF (NA of 0.2) and (c) a 100/140 $\mu \mathrm{m}$ MMF (NA of 0.29) input. The launches used range from restricted (4 $\mu \mathrm{m}$ SMF) to relatively overfilled $(100 \mu \mathrm{m} \mathrm{MMF})$.

The experimental setup used is shown in Fig. 6. Both ends of the flexible waveguide samples are mounted on translation stages and the sample is tightly wrapped $180^{\circ}$ around a cylindrical mandrel of constant radius. A pair of microscope objectives is used to couple the light emitted from an $850 \mathrm{~nm}$ multimode VCSEL into a fibre patchcord of the appropriate type while a cleaved fibre of the same type is used to couple the light into the waveguide under test. Index matching gel is used at the waveguide input to minimise coupling losses and any scattering due to facet surface roughness.

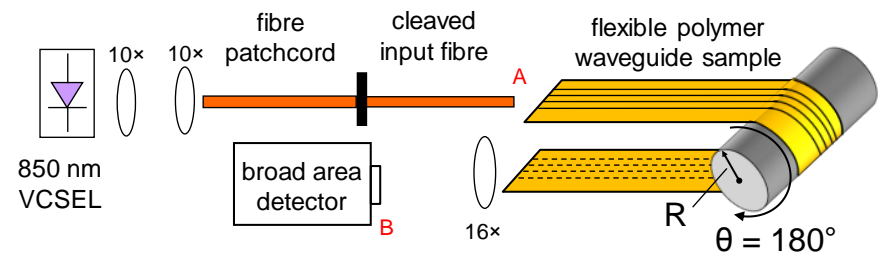

Fig. 6. Experimental setup used for the bending loss measurements.

Prior to the characterisation of the waveguide samples, the far- and near- field profile of the beam emitted from the cleaved fibre end are recorded for the different launches.
Fig. 7(a) shows the normalised far-field profile, while Fig. 7(b) the near-field images of the fibre end. The red line in Fig. 7 (a) indicates the $5 \%(-13 \mathrm{~dB})$ far-field angle (FFA) which approximates the NA of the fibre [33] and it is found to be $7^{\circ}$, $10.25^{\circ}$ and $16^{\circ}$ for the $4 \mu \mathrm{m} \mathrm{SMF}, 50 \mu \mathrm{m} \mathrm{MMF}$ and $100 \mu \mathrm{m}$ MMF inputs respectively. The calculated 5\% FFA value using the nominal fibre NA is $7.5^{\circ}, 11.5^{\circ}$ and $16.9^{\circ}$ for the $4 \mu \mathrm{m}$ $\mathrm{SMF}, 50 \mu \mathrm{m} \mathrm{MMF}$ and $100 \mu \mathrm{m}$ MMF input respectively. The acceptance angle of the waveguide $\left(\mathrm{AA}_{\mathrm{wg}}\right)$ is calculated to be $12.4^{\circ}$. The far-field measurements confirm the characterisation of the $4 \mu \mathrm{m} \mathrm{SMF}, 50 \mu \mathrm{m}$ MMF and $100 \mu \mathrm{m}$ MMF inputs as restricted $\left(\mathrm{FFA}<\mathrm{AA}_{\mathrm{wg}}\right)$, typical $\left(\mathrm{FFA} \sim \mathrm{AA}_{\mathrm{wg}}\right)$ and relatively overfilled $\left(\mathrm{FFA}>\mathrm{AA}_{\mathrm{wg}}\right)$ launch respectively.

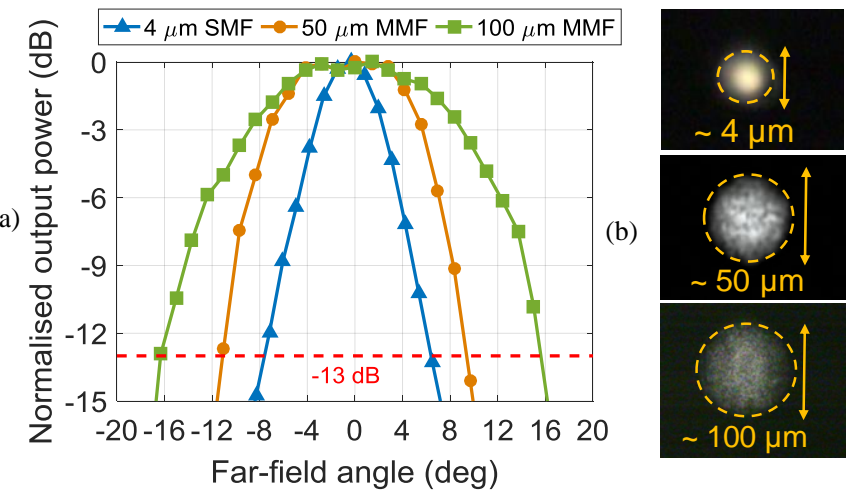

Fig. 7. (a) Normalised far-field profile and (b) near-field images of the fibres used in the characterisation of the flexible waveguide samples. The red dashed line in (a) indicates the $5 \%$ value $(-13 \mathrm{~dB})$.

At the waveguide output, a microscope objective $(\times 16)$ with an NA (0.32) larger than that of the waveguide (NA of $~ 0.21$ ) is used to collect the emitted light and focus it onto a broad area photodetector. The insertion loss (difference in received power between points A and B in Fig. 6) of each waveguide on the flexible sample is recorded and the average value is obtained. Mandrels of different diameter are used to obtain the variation of the insertion loss as a function of the bending radius. Additionally, due to the asymmetry of the sample cross section for the new waveguide design, the measurement is carried out when the samples are bent in both out-of-plane directions: towards the top (air) and bottom (substrate) (Fig. 8). The average insertion loss of the waveguides is also measured using the same setup and launch condition when no flexure is applied (straight samples, Fig. 9) and is compared with the value obtained under flexure to obtain the excess bending loss for each bending direction and launch (Fig. 10).

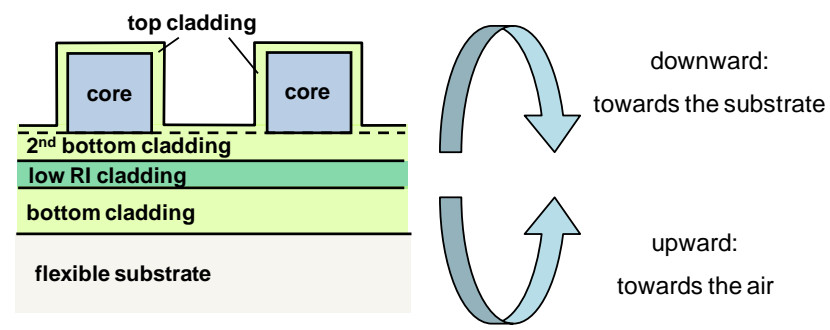

Fig. 8. Illustration of the sample bending directions out-of-plane to the waveguide array. 


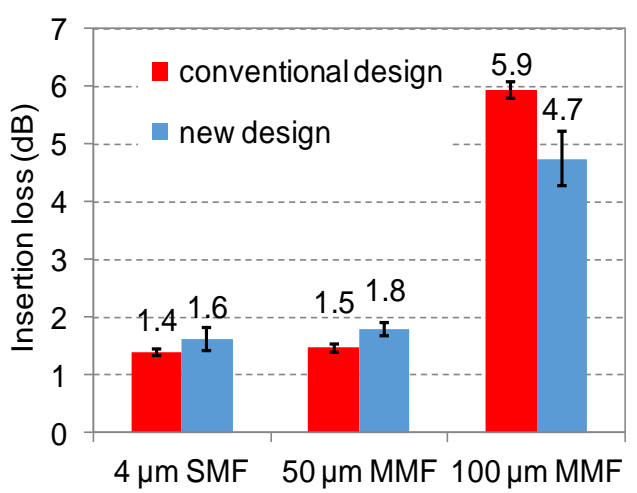

Fig. 9. Average insertion loss for the two flexible waveguide samples when no flexure is applied (straight samples). Error bars indicate one standard deviation of the measured values. (a)

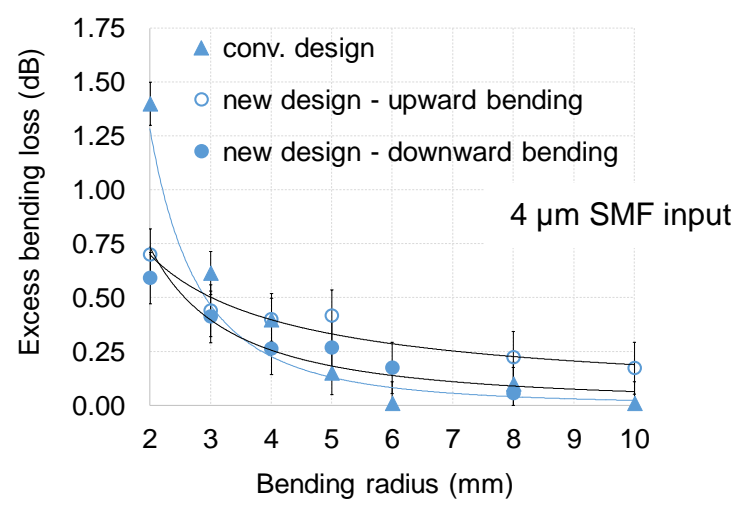

(b)

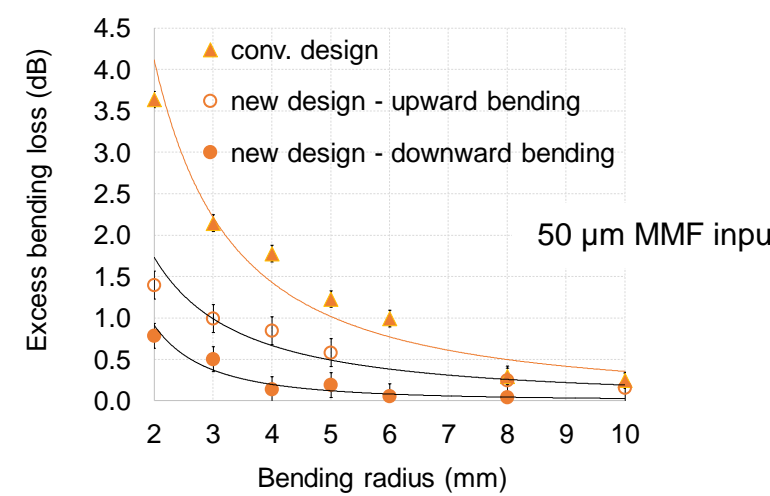

Fig. 10. Excess bending loss for the flexible waveguide samples with the conventional (triangle) and new design (circles) for the different launch conditions employed: (a) $4 \mu \mathrm{m} \mathrm{SMF}$, (b) $50 \mu \mathrm{m}$ MMF and (c) $100 \mu \mathrm{m} \mathrm{MMF}$. The filled circles correspond to downward bending (towards the substrate), while the un-filled ones to upward bending (towards the air).

The insertion loss values obtained for the straight samples (Fig. 9) indicate similar loss performance from the waveguides featuring the two designs when no flexure is applied. The loss measurements under flexure (Fig. 10) demonstrate however that a significant improvement in bending loss performance can be achieved with the proposed waveguide design. This improvement is more pronounced for the more overfilled launch with the $100 \mu \mathrm{m}$ MMF input as larger percentage of power is coupled to the higher order modes which are more susceptible to bending loss. The excess bending loss for a $3 \mathrm{~mm}$ radius is reduced from $6.5 \mathrm{~dB}$ for the conventional design under the $100 \mu \mathrm{m}$ MMF launch to 2.8 and $2.4 \mathrm{~dB}$ for the new design under the same launch for upward and downward bending respectively. As expected, downward bending (towards the substrate) yields a lower bending loss due to the larger difference between the core RI and the air on the outside of the bend. Similarly, for the same $3 \mathrm{~mm}$ radius and the $50 \mu \mathrm{m}$ MMF input, the excess bending loss for the new design is found to be 1 and $0.5 \mathrm{~dB}$ for upward and downward bending respectively. This is an improvement of 1.2 and $1.7 \mathrm{~dB}$ over the waveguides with the conventional design ( $2.2 \mathrm{~dB}$ of excess loss at $3 \mathrm{~mm}$ radius) under the same launch. Radii as low as $2 \mathrm{~mm}$ can be used with an excess loss below $1 \mathrm{~dB}$ for a $50 \mu \mathrm{m}$ MMF input which matches typical launches in real-world systems. For the $4 \mu \mathrm{m}$ SMF input, the observed differences are much smaller, as most of the power is coupled to lower order modes which are less susceptible to bending loss. The loss reduction is found to be $\sim 0.2 \mathrm{~dB}$ for the $3 \mathrm{~mm}$ radius and for both bending directions.

\section{TWISTING LOSS PERFORMANCE}

The twisting loss performance of flexible samples depends on the sample dimensions (length, width and thickness), applied force and twisting turns. As a result, the excess loss due to twisting of polymer waveguides on 3 flexible samples with different dimensions is assessed: (i) sample $\mathrm{A}$, $135 \times 10 \times 0.2 \mathrm{~mm}^{3}$, (ii) sample B, $242 \times 6 \times 0.2 \mathrm{~mm}^{3}$ and (iii) sample C, $242 \times 2 \times 0.2 \mathrm{~mm}^{3}$. Sample A is the shorter and wider sample, samples $\mathrm{B}$ and $\mathrm{C}$ are considerably longer, while sample $\mathrm{C}$ is additionally very narrow. Images of the 3 flexible samples are shown in Fig. 11. All samples used in this study are based on the conventional design [Fig. 1(a)] as the fabricated samples with new proposed design are too short to carry out proper twisting measurements. However, it should be noted that we believe that the general observations and discussion presented in this section are also valid for flexible waveguide samples with the new design.

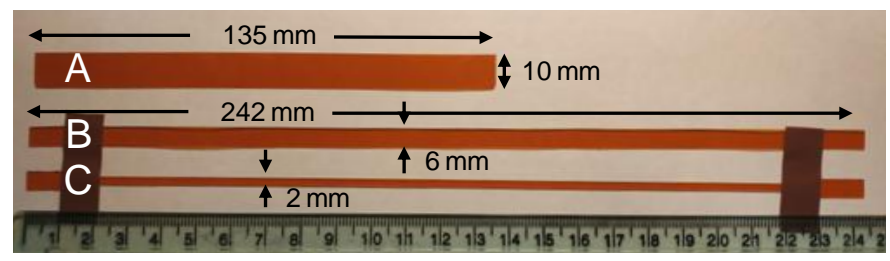

Fig. 11. Images of the flexible waveguide samples used in the twisting loss studies: sample A, $135 \times 10 \times 0.2 \mathrm{~mm}^{3}$, sample B, $242 \times 6 \times 0.2 \mathrm{~mm}^{3}$ and sample C, $242 \times 2 \times 0.2 \mathrm{~mm}^{3}$.

The experimental setup for the twisting loss measurements is shown in Fig. 12. The two ends of the flexible samples are clamped on sub-mounts and positioned on translation stages. One sample end is in-plane rotated a given angle, twisting the 
sample. 1 full twisting turn corresponds to a $360^{\circ}$ rotation of the sample end. The sample is stretched as much as possible without damaging the waveguides in order to approximate the sample length without any twisting applied. Sample images of the flexible samples twisted different number of full turns are shown in Fig. 13. The same launch conditions as the ones employed in the bending loss measurements are also used here. The excess twisting loss of the waveguides is obtained by comparing the insertion loss of the waveguides with that obtained when no twisting applied (straight sample) under the same launch condition.

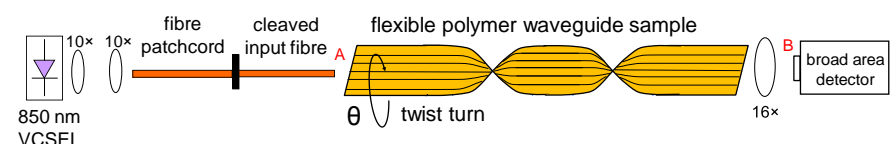

Fig. 12. Experimental setup for the twisting loss measurements.

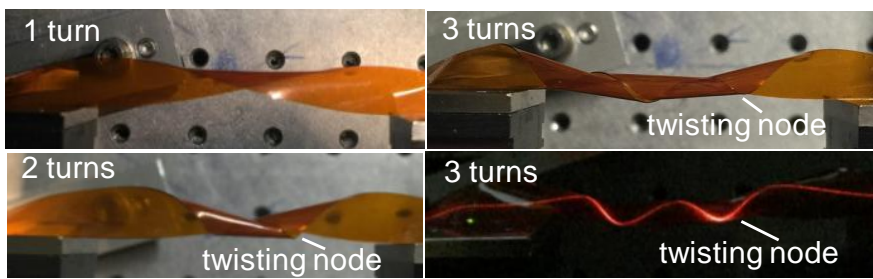

(a)

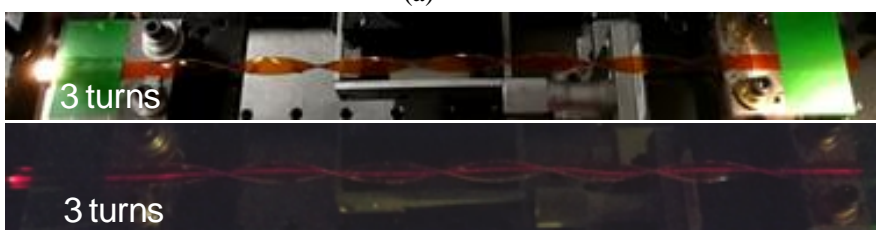

(b)

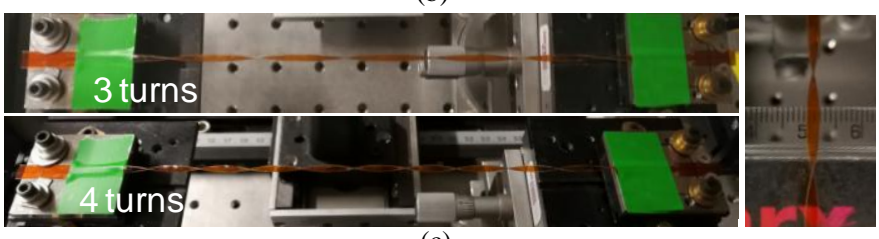

(c)

Fig. 13. Images of the flexible waveguide samples twisted different number of turns: (a) sample A, (b) sample B and (c) sample C.

Fig. 14 shows the obtained excess twisting loss for the different samples. It can be noticed that twisting sample A results in low excess loss provided that the twisting turns applied are less than two. The increase in loss is due to the formation of a twisting node (self-contact) in the sample when two or more twisting turns are applied [Fig. 13(a)]. This node essentially causes tight bending of the waveguides over this area resulting in the increase in excess loss. The image of one waveguide in sample A twisted 3 full turns and illuminated with red light in Fig. 13(a) clearly shows the higher scattering loss in the twisting node area. Once the node is generated, the further application of more twisting turns does not result in any significant change in loss as the node remains un-affected.

On the contrary, samples B and C do not exhibit this sudden increase in loss as no twisting node is formed when up to 4 full twisting turns are applied. For sample B, the excess twisting loss gradually increases with increasing number of full turns, but remains low $<0.8 \mathrm{~dB}$ even for the relatively overfilled launch with the $100 \mu \mathrm{m}$ MMF. For the $50 \mu \mathrm{m}$ MMF input which better matches typical launch conditions in real- world systems, the observed excess loss is below $0.3 \mathrm{~dB}$ for all number of turns applied. For sample $\mathrm{C}$, which is narrower, the twisting loss is found to be negligible $(<0.15 \mathrm{~dB})$ for the SMF and MMF inputs for all twisting turns applied. The results indicate that, in practice, twisting of sample $\mathrm{C}$ has no significant effect on its light propagation characteristics.

(a)
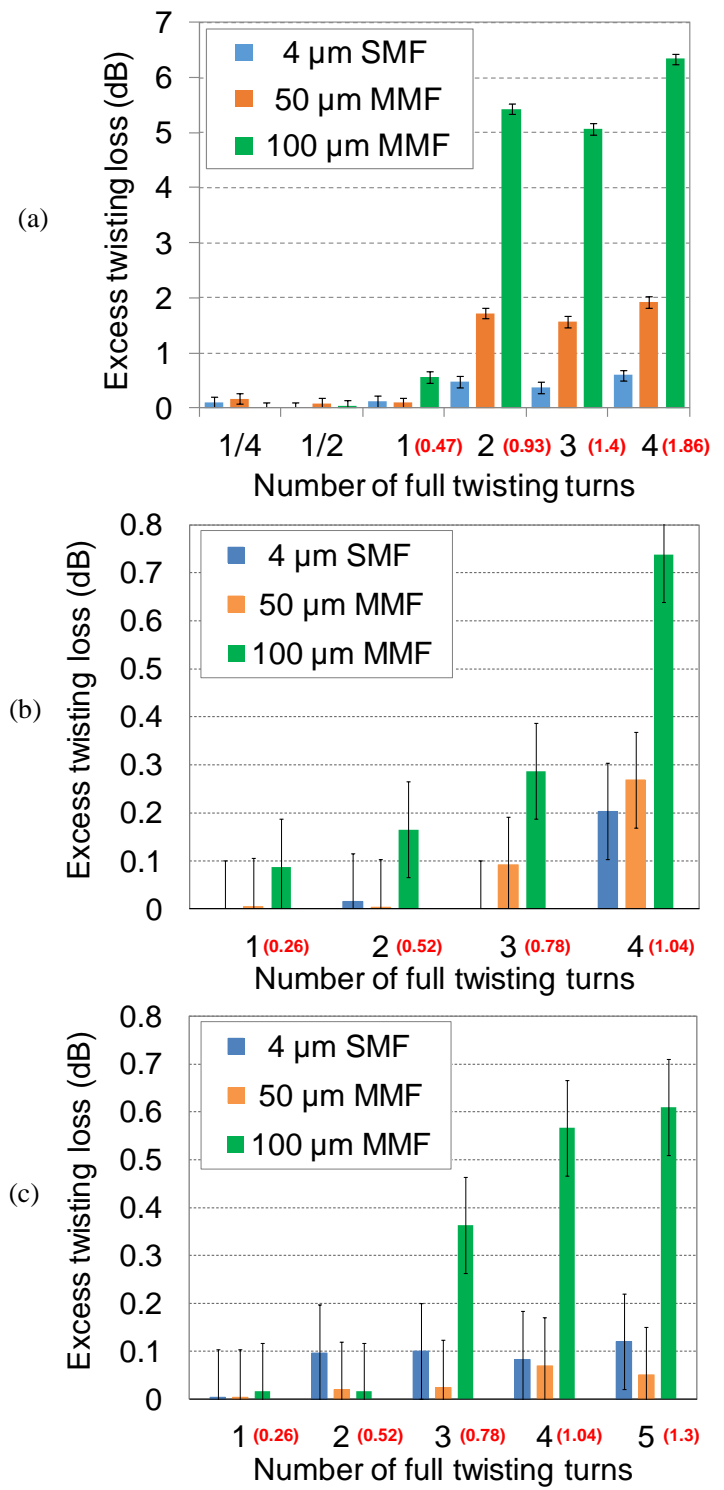

Fig. 14. Excess twisting loss for the 3 flexible waveguide samples under the 3 launch conditions studied: (a) sample A, $135 \times 10 \times 0.2 \mathrm{~mm}^{3}$, (b) sample B, $242 \times 6 \times 0.2 \mathrm{~mm}^{3}$ and (c) sample C, $242 \times 3 \times 0.2 \mathrm{~mm}^{3}$. In brackets, the corresponding normalised twisting angle per length $a=\theta / L$ (in $\mathrm{rad} / \mathrm{cm}$ ) is also provided for each sample for the different number of twisting turns applied.

This behaviour can be understood by considering the different forms of flexible ribbons that are generated when twisting is applied $[34,35]$. These include helicoids, wrinkles and loops (nodes) and depend on the geometric parameters of the samples and the applied longitudinal force [Fig. 15(a)]. Using the equations presented in [35], phase diagrams can be generated that relate the shape produced with the applied force and sample rotation (twisting turns) [Fig. 15(b)]. The phase diagrams for the flexible samples A, B and C have been calculated and are shown in Fig. 16. With regards to the 
twisting loss performance, the results presented above indicate that no significant excess loss is induced in the flexible samples provided that the formation of self-contact node is avoided. As a result, it is adequate to examine the limit of selfcontact behaviour for the flexible waveguides samples. The red line in Fig. 16 indicates the delineation between the selfcontact area (i.e. twisting node, above the line) and different types of helicoids (below the line). For relatively long and thin ribbons such as the ones used here, the normalised delineation angle $\theta_{d l}$ (as a number of full twisting turns) can be estimated using the equation [34]:

$$
\theta_{d l}=\left(\frac{t}{w}\right)^{3 / 2}\left(\frac{Y}{F}\right)^{1 / 2} \frac{L}{2 \pi}
$$

where $L, w, t$ are the length, width and thickness of the sample respectively, $Y$ is its Young's modulus and $F$ is applied longitudinal force. For the polymer materials used in this work, the Young's modulus is $2.77 \mathrm{GPa}$.
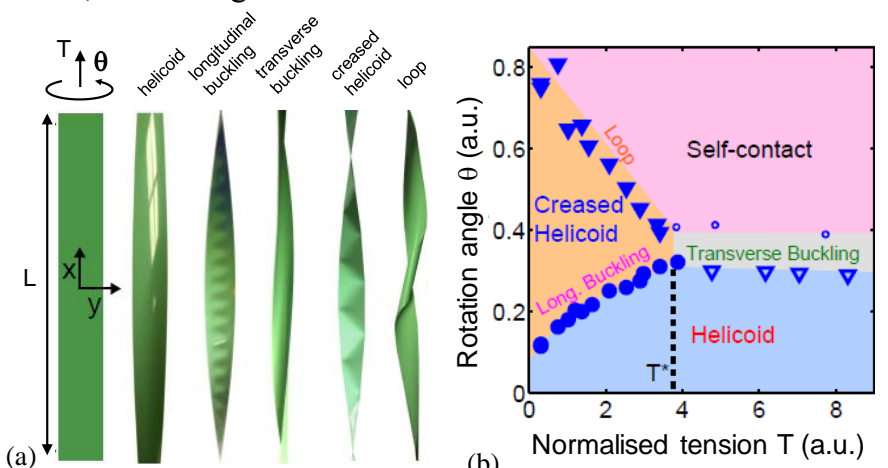

Fig. 15. (a) Different forms of a flexible ribbon generated through twisting and (b) sample phase diagram indicating the different forms generated as a function of the longitudinal tension and rotation applied. Reproduced from [35] with permission.
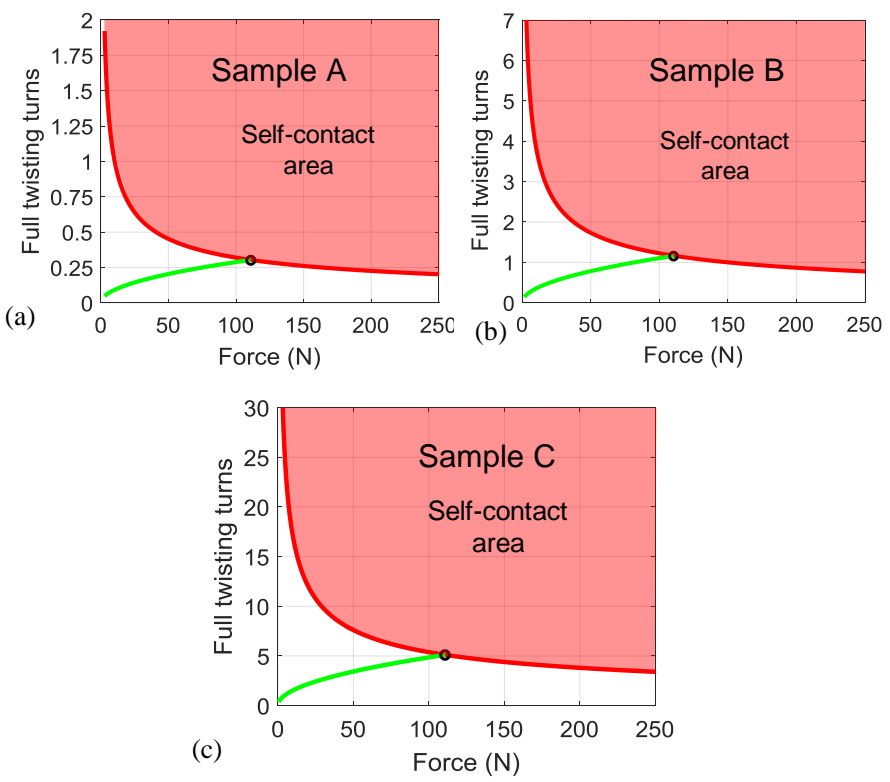

Fig. 16. Calculated phase diagrams for the 3 flexible waveguide samples: (a) sample A, $135 \times 10 \times 0.2 \mathrm{~mm}^{3}$, (b) sample B, $240 \times 6 \times 0.2 \mathrm{~mm}^{3}$ and (c) sample C, $240 \times 3 \times 0.2 \mathrm{~mm}^{3}$. The red line and shaded area indicate the delineation angle $\theta_{d l}$ and self-contact area respectively for each sample.

It can be noticed from the phase diagram of sample A [Fig. 16(a)] that for more than 1.5 twisting turns, self-contact occurs irrespective of the applied force. The use of narrower samples (B and C) allows much larger number of full twisting turns to be applied before the formation of the twisting node. The maximum number of full twisting turns is estimated to approximately 5 and 20 for samples B and C respectively. The obtained values are in good agreement with the experimental observations. As a result, eq. (1) can provide a useful practical rule for examining the twisting limit of flexible polymer waveguide samples when used in real-world systems.

\section{CONCLUSIONS}

Flexible polymer waveguides can provide high-speed shortreach optical interconnection in chip-to-chip communications and in in-car and in-plane networks. Their practical use however is limited by their ability to withstand out-of-plane bending and in-plane twisting without severe degradation of their loss performance. In this work therefore, we propose the use of a new waveguide design that provides similar loss performance with the conventional waveguide design when no flexure is applied but an improved bending loss performance under flexure. In addition, a suitable design rule that ensures low twisting loss for flexible waveguide samples is derived.

The proposed new waveguide design can be readily produced with the conventional fabrication methods of such waveguides, and it is experimentally shown that it can offer significant improvement in bending loss performance over the conventional design. For example, a $0.5 \mathrm{~dB}$ excess bending loss is achieved for a $3 \mathrm{~mm}$ radius under a $50 \mu \mathrm{m}$ MMF launch which constitutes a $1.7 \mathrm{~dB}$ improvement over the conventional waveguide for the same radius and launch condition. With respect to twisting loss performance, it is experimentally shown that avoiding the formation of nodes in flexible polymer samples due to twisting is sufficient to ensure low excess loss. A practical rule associating the samples' geometrical parameter and applied tension is provided that allows the estimation of this twisting limit for practical applications.

\section{REFERENCES}

[1] D. A. B. Miller, "Rationale and challenges for optical interconnects to electronic chips," Proceedings of the IEEE, vol. 88, pp. 728-749, 2000.

[2] M. A. Taubenblatt, "Optical Interconnects for High-Performance Computing," Journal of Lightwave Technology, vol. 30, pp. 448-457, 2012.

[3] F. E. Doany, C. L. Schow, B. G. Lee, R. A. Budd, C. W. Baks, C. K. Tsang, J. U. Knickerbocker, R. Dangel, B. Chan, L. How, C. Carver, H. Jianzhuang, J. Berry, D. Bajkowski, F. Libsch, and J. A. Kash, "Terabit/s-Class Optical PCB Links Incorporating 360-Gb/s Bidirectional $850 \mathrm{~nm}$ Parallel Optical Transceivers," Journal of Lightwave Technology, vol. 30, pp. 560-571, 2012.

[4] R. Dangel, C. Berger, R. Beyeler, L. Dellmann, M. Gmur, R. Hamelin, F. Horst, T. Lamprecht, T. Morf, S. Oggioni, M. Spreafico, and B. J. Offrein, "Polymer-Waveguide-Based Board-Level Optical Interconnect Technology for Datacom Applications," IEEE Transactions on Advanced Packaging, vol. 31, pp. 759-767, 2008.

[5] N. Bamiedakis, J. Beals, R. V. Penty, I. H. White, J. V. DeGroot, and T. V. Clapp, "Cost-Effective Multimode Polymer Waveguides for HighSpeed On-Board Optical Interconnects," IEEE Journal of Quantum Electronics, vol. 45, pp. 415-424, 2009.

[6] M. Immonen, J. Wu, H. J. Yan, L. X. Zhu, P. Chen, and T. RapalaVirtanen, "Development of electro-optical PCBs with embedded waveguides for data center and high performance computing applications," Proc. SPIE 8991, Optical Interconnects XIV, vol. 8991, pp. 1-9, 2014.

[7] N. Bamiedakis, A. Hashim, J. Beals, R. V. Penty, and I. H. White, "Low-Cost PCB-Integrated 10-Gb/s Optical Transceiver Built With a 
Novel Integration Method," IEEE Transactions on Components, Packaging and Manufacturing Technology, vol. 3, pp. 592-600, 2013.

[8] M. Immonen, R. Zhang, M. Press, H. Tang, W. Lei, J. Wu, H. J. Yan, L. X. Zhu, and M. Serbay, "End-to-end Optical 25Gb/s Link Demonstrator with Embedded Waveguides, 90o Out-of-Plane Connector and Onboard Optical Transceivers," in 42nd European Conference on Optical Communication (ECOC), 2016, pp. 1154-1156.

[9] R. Kinoshita, K. Moriya, K. Choki, and T. Ishigure, "Polymer Optical Waveguides With GI and W-Shaped Cores for High-Bandwidth-Density On-Board Interconnects," Journal of Lightwave Technology, vol. 31, pp. 4004-4015, 2013.

[10] K. Schmidtke, F. Flens, A. Worrall, R. Pitwon, F. Betschon, T. Lamprecht, and R. Krahenbuhl, "960 Gb/s Optical Backplane Ecosystem Using Embedded Polymer Waveguides and Demonstration in a 12G SAS Storage Array," Journal of Lightwave Technology, vol. 31, pp. 3970-3975, 2013.

[11] R. C. A. Pitwon, W. Kai, J. Graham-Jones, I. Papakonstantinou, H. Baghsiahi, B. J. Offrein, R. Dangel, D. Milward, and D. R. Selviah, "FirstLight: Pluggable Optical Interconnect Technologies for Polymeric Electro-Optical Printed Circuit Boards in Data Centers," Journal of Lightwave Technology, vol. 30, pp. 3316-3329, 2012.

[12] N. Bamiedakis, A. Hashim, R. V. Penty, and I. H. White, "A 40 Gb/s Optical Bus for Optical Backplane Interconnections," Journal of Lightwave Technology, vol. 32, pp. 1526-1537, 2014.

[13] J. Beals, N. Bamiedakis, A. Wonfor, R. Penty, I. White, J. DeGroot, K. Hueston, T. Clapp, and M. Glick, "A terabit capacity passive polymer optical backplane based on a novel meshed waveguide architecture," Applied Physics A: Materials Science \& Processing, vol. 95, pp. 983988, 2009.

[14] R. Dangel, J. Hofrichter, F. Horst, D. Jubin, A. La Porta, N. Meier, I. M. Soganci, J. Weiss, and B. J. Offrein, "Polymer waveguides for electrooptical integration in data centers and high-performance computers," Optics Express, vol. 23, pp. 4736-4750, 2015.

[15] J. Chen, N. Bamiedakis, P. P. Vasil'ev, T. J. Edwards, C. T. A. Brown, R. V. Penty, and I. H. White, "High-Bandwidth and Large Coupling Tolerance Graded-Index Multimode Polymer Waveguides for On-Board High-Speed Optical Interconnects," Journal of Lightwave Technology, vol. 34, pp. 2934-2940, 2016.

[16] J. Chen, N. Bamiedakis, T. J. Edwards, C. T. A. Brown, R. V. Penty, and I. H. White, "Dispersion studies on multimode polymer spiral waveguides for board-level optical interconnects," in IEEE Optical Interconnects Conference (OIC), 2015, pp. 26-27.

[17] N. Bamiedakis, J. Chen, P. Westbergh, J. S. Gustavsson, A. Larsson, R. V. Penty, and I. H. White, "40 Gb/s Data Transmission Over a 1-mLong Multimode Polymer Spiral Waveguide for Board-Level Optical Interconnects," Journal of Lightwave Technology, vol. 33, pp. 882-888, 2015.

[18] N. Bamiedakis, J. Wei, J. Chen, P. Westbergh, A. Larsson, R. V. Penty, and I. H. White, "56 Gb/s PAM-4 Data Transmission Over a $1 \mathrm{~m}$ Long Multimode Polymer Interconnect," in Conference on Lasers and Electro-Optics (CLEO) San Jose, California: Optical Society of America, 2015, p. STu4F.5.

[19] H. Zuo, S. Yu, T. Gu, and J. Hu, "Low loss, flexible single-mode polymer photonics," Optics Express, vol. 27, pp. 11152-11159, 2019.

[20] F. Shi, N. Bamiedakis, P. P. Vasil'ev, R. V. Penty, I. H. White, and D. Chu, "Flexible Multimode Polymer Waveguide Arrays for Versatile High-Speed Short-Reach Communication Links," Journal of Lightwave Technology, vol. 36, pp. 2685-2693, 2018.

[21] K. Nieweglowski, L. Lorenz, S. Lungen, T. Tiedje, K.-J. Wolter, and K. Bock, "Optical coupling with flexible polymer waveguides for chip-tochip interconnects in electronic systems," Microelectronics Reliability, vol. 84, pp. 121-126, 2018.

[22] R. Dangel, F. Horst, D. Jubin, N. Meier, J. Weiss, B. J. Offrein, B. W. Swatowski, C. M. Amb, D. J. DeShazer, and W. K. Weidner, "Development of Versatile Polymer Waveguide Flex Technology for Use in Optical Interconnects," Journal of Lightwave Technology, vol. 31, pp. 3915-3926, 2013.

[23] H. Sung Hwan, L. Jung Woon, L. Woo-Jin, K. Gye Won, C. Che Hyun, A. Jong Bea, J. Ki Young, C. Kyung Soon, and R. Byung Sup, "Bendable and Splitter-Integrated Optical Subassembly Based on a Flexible Optical Board," IEEE Photonics Technology Letters, vol. 22, pp. 167-169, 2010.

[24] L. Li, Y. Zou, H. Lin, J. Hu, X. Sun, N.-N. Feng, S. Danto, K. Richardson, T. Gu, and M. Haney, "A Fully-Integrated Flexible
Photonic Platform for Chip-to-Chip Optical Interconnects," Journal of Lightwave Technology, vol. 31, pp. 4080-4086, 2013.

[25] G. Jiang, S. Baig, and M. R. Wang, "Flexible Polymer Waveguides With Integrated Mirrors Fabricated by Soft Lithography for Optical Interconnection," Journal of Lightwave Technology, vol. 31, pp. 1835$1841,2013$.

[26] H. Numata, M. Tokunari, and J. B. Heroux, "60-Micrometer pitch polymer waveguide array attached active optical flex," in Optical Fiber Communications Conference and Exhibition (OFC), 2017, pp. 1-3.

[27] X. Dou, X. Wang, X. Lin, D. Ding, D. Z. Pan, and R. T. Chen, "Highly flexible polymeric optical waveguide for out-of-plane optical interconnects," Optics Express, vol. 18, pp. 16227-16233, 2010.

[28] L. Li, H. Lin, J. Michon, S. Geiger, J. Li, H. Zheng, Y. Huang, A. Yadav, K. A. Richardson, and T. Gu, "Mechanically Flexible Integrated Photonic Systems for Sensing and Communications," ECS Transactions, vol. 77, pp. 37-46, 2017.

[29] R. S. E. John, C. M. Amb, B. W. Swatowski, W. K. Weidner, M. Halter, T. Lamprecht, and F. Betschon, "Thermally Stable, Low Loss Optical Silicones: A Key Enabler for Electro-Optical Printed Circuit Boards," Journal of Lightwave Technology, vol. 33, pp. 814-819, 2015.

[30] B. W. Swatowski, C. M. Amb, S. K. Breed, D. J. Deshazer, W. K. Weidner, R. F. Dangel, N. Meier, and B. J. Offrein, "Flexible, stable, and easily processable optical silicones for low loss polymer waveguides," Proc. SPIE 8622, Organic Photonic Materials and Devices $X V$, pp. 1-11, 2013.

[31] A. Boersma, S. Wiegersma, B. J. Offrein, J. Duis, J. Delis, M. Ortsiefer, G. van Steenberge, M. Karpinen, A. van Blaaderen, and B. Corbett, "Polymer-based optical interconnects using nanoimprint lithography," in Proc. SPIE 8630, Optoelectronic Interconnects XIII, 2013, pp. 1-9.

[32] J. C. Dingeldein, K. A. Walczak, B. W. Swatowski, C. R. Friedrich, C. T. Middlebrook, and M. C. Roggemann, "Process characterization for direct dispense fabrication of polymer optical multi-mode waveguides," Journal of Micromechanics and Microengineering, vol. 23, p. 075015, 2013.

[33] ANSI/TIA-455-177-B standard: FOTP-177, IEC-60793-1-43 Optical Fibres - Part 1-43: Measurement Methods and Test Procedures Numerical Aperture, 2003

[34] J. Chopin, V. Demery, and B. Davidovitch, "Roadmap to the Morphological Instabilities of a Stretched Twisted Ribbon," Journal of Elasticity, vol. 119, pp. 137-189, 2015.

[35] J. Chopin and A. Kudrolli, "Helicoids, Wrinkles, and Loops in Twisted Ribbons," Physical Review Letters, vol. 111, p. 174302, 2013. 